\title{
A STUDY ON DETECTION OF GLYCOPEPTIDE RESISTANCE AMONG CLINICAL ISOLATES OF COAGULASE NEGATIVE STAPHYLOCOCCUS (CONS) SPECIES IN A TERTIARY CARE CENTRE
}

\author{
S. Meerah'1, P. A. T. Jagatheeswary²
}

${ }_{1}^{1}$ Assistant Professor, Department of Microbiology, Chengalpattu Medical College, Chengalpattu, Tamilnadu.

2Professor, Department of Microbiology, Saveetha Medical College, Thandalam, Chennai, Tamilnadu.

ABSTRACT
BACKGROUND
CoNS have become recognised as important agents of hospital acquired infections. Infections with CoNS occur mainly in patients
with indwelling foreign devices, neonates and in immunocompromised patients. Some recent studies have pointed out reduced
susceptibility of CoNS to Glycopeptide antibiotics.
In view of the above perspective, the present study was carried out in a tertiary care hospital in Madurai to assess the
prevalence of Glycopeptide resistant CoNS species in our region and also to assess the antibiotic of choice for them.

\section{MATERIALS AND METHODS}

The present study was a cross-sectional study conducted in Institute of Microbiology, Madurai Medical College, Madurai. The study period was from November 2014 to August 2015. A total of 104 CoNS species were isolated from clinical samples, collected from the patients admitted in various wards of Government Rajaji Hospital, Madurai and processed using conventional microbiological methods.

\section{RESULTS}

Among the 104 CoNS species 72 were S. epidermidis, 19 were S. lugdunensis, 11 were S. haemolyticus and 2 were S. saprophyticus. Among them, 28 (26.92\%) were Methicillin Resistant CoNS (MRCoNS) and 76 (73.08\%) were Methicillin Sensitive CoNS (MSCoNS). Phenotypic detection of Glycopeptide resistance among Methicillin Resistant Staphylococci by various methods showed one isolate 3.6\% (among MRCoNS) to be Vancomycin Intermediate S. epidermidis (VISE) from blood sample. This isolate was susceptible to Gentamicin, Teicoplanin, Linezolid, Rifampicin, Quinupristin/ Dalfopristin by reference CLSI methods.

\section{CONCLUSION}

Early detection of these strains is crucial to establish an appropriate antimicrobial therapy, thereby reducing the mortality and morbidity associated with these infections and also to prevent hospital acquired infections.

\section{KEYWORDS}

Glycopeptide Resistance, Indwelling Foreign Devices, Immunocompromised.

HOW TO CITE THIS ARTICLE: Meerah S, Jagatheeswary PAT. A study on detection of glycopeptide resistance among clinical isolates of coagulase negative staphylococcus (CoNS) species in a tertiary care centre. J. Evolution Med. Dent. Sci. 2018;7(07):829833, DOI: $10.14260 /$ jemds/2018/189

\section{BACKGROUND}

CoNS have become recognised as important agents of hospital acquired infections. Infections with CoNS occur mainly in patients with indwelling foreign devices, neonates and in immunocompromised patients. The pathogenicity of CoNS species might be due to the production of slime that helps the organism to adhere to pacemaker, prosthetic heart valves, smooth plastic surfaces and prosthetic devices of joints. Over the past decades, Methicillin resistance in CoNS has substantially increased and has led to the use of Glycopeptides like Vancomycin and Teicoplanin. Some recent studies have pointed out reduced susceptibility of CoNS to Glycopeptide antibiotics.

'Financial or Other Competing Interest': None.

Submission 03-01-2018, Peer Review 31-01-2018,

Acceptance 05-02-2018, Published 12-02-2018.

Corresponding Author:

Dr. P. A. T. Jagatheeswary,

Professor, Department of Microbiology,

Saveetha Medical College,

Thandalam, Chennai-602105,

Tamilnadu, India.

E-mail: patjags@rediffmail.com

DOI: 10.14260/jemds/2018/189

\section{(c) $(i) \Theta$}

It is an essential part of the surveillance system of each and every hospital setup to monitor continuously such Glycopeptide resistant CoNS infections, and to assess the antibiotic susceptibility pattern of them. In view of the above perspective, the present study was carried out in our tertiary care hospital in Madurai to assess the prevalence of Glycopeptide resistant CoNS species in our region and also to assess the antibiotic of choice for them.

\section{Aims and Objectives}

- To isolate and speciate Coagulase Negative Staphylococci (CoNS) from various clinical samples of inpatients admitted at GRH, Madurai.

- To determine the distribution of Methicillin resistance among the isolated CoNS species.

- To study the prevalence of Glycopeptide resistance among the isolated Methicillin Resistant CoNS species by various phenotypic methods.

\section{MATERIALS AND METHODS}

The present study was a cross-sectional study conducted in Institute of Microbiology, Madurai Medical College, Madurai. The study period was from November 2014 to August 2015. Ethical committee clearance from the institution was obtained and informed written consent was received from 
the patients before collecting the specimens. A total of 104 CoNS species were isolated from clinical samples, collected from the patients admitted in various wards of Government Rajaji Hospital, Madurai and processed using conventional microbiological methods.

Speciation of CoNS was done based on Kloos and Schleifer classification and Koneman(1,2) by studying the colony morphology on nutrient agar, Gram reaction of smear, Haemolysis on blood agar, Mannitol salt agar, Catalase test, Coagulase test, Modified oxidase test, nitrate reduction test, Voges-Proskauer test, Urease test, Oxidative fermentative test, DNAse test, Phosphatase test, Ornithine decarboxylase, Sugar fermentation-Glucose, lactose, Mannitol, Mannose, Trehalose, Xylose, Novobiocin and Polymyxin B 300 Susceptibility test. Antimicrobial susceptibility testing was done based on CLSI Guidelines.

Identification of MRCONS: (Antibiogram by Kirby-Bauer Disk Diffusion Method) ${ }^{(3)}$

All isolates of CoNS were tested by using 30 micrograms of cefoxitin by Disk Diffusion method for Methicillin sensitivity and interpretation was done as per CLSI Guidelines. ATCC S.aureus 25923 was used as negative control (Cefoxitin 30 microgram Zone size 23 - $29 \mathrm{~mm}$ ). ATCC S. aureus 43300 as positive control with (Zone size $\leq 21 \mathrm{~mm}$ ).

Phenotypic Detection of Glycopeptide Resistance among Methicillin Resistant Coagulase Negative Staphylococci $(n=28)$ was analysed.

\section{Vancomycin Screen Agar (BHIA6 $\mu$ g V) (3)}

All the Methicillin Resistant CoNS isolates were screened for vancomycin resistance by Vancomycin screen agar, that is brain heart infusion (BHI) agar containing $6 \mu \mathrm{g} / \mathrm{mL}$ of Vancomycin and is used for the presumptive identification of vancomycin resistance. Spot inoculation of $10 \mu \mathrm{L}$ of 0.5 McFarland turbidity standard bacterial suspension along with positive and negative control strains was done onto the agar surface. The plates were incubated for $24 \mathrm{hrs}$. aerobically at $37^{\circ} \mathrm{C}$. Growth of greater than 1 colony indicates presumptive vancomycin resistance. It should be confirmed by determining the minimum inhibitory concentration (MIC) for vancomycin and teicoplanin. This test includes $\mathrm{E}$. faecalis ATCC 29212 as negative control (MIC-Vancomycin $\leq 4$ microgram $/ \mathrm{mL}$ ), E. faecalis ATCC 51299 as positive control (MIC-Vancomycin $\geq 8$ microgram $/ \mathrm{mL}$ ).

E-Test Method: (The Vancomycin and Teicoplanin Minimum Inhibitory Concentration (MIC) for all the Methicillin Resistant CoNS Isolates were tested by E-Test Method)

Preparation of inoculum and the test procedures were done as per the instruction given by the manufacturer (Hi-Media). Interpretation of results were done according to CLSI 2014 guidelines.

MIC reading was done as per manufacturer's instructions. Read the MIC where the ellipse intersects the MIC scale on the strip. For bactericidal drugs such as Vancomycin, Teicoplanin always read the MIC at the point of complete inhibition of all growth including hazes, microcolonies and isolated colonies, if necessary use magnifying glass. Always round up the value to the next two-fold dilution before categorisation.

\section{Quality Control Strains}

ATCC 29213 S. aureus with Vancomycin MIC $\leq 2$ microgram/mL and ATCC 700699 (MU50) VISA strains with Vancomycin MIC 4 - 8 microgram $/ \mathrm{mL}$ were used.

\section{BROTH MICRODILUTION METHOD (BMD)}

(The vancomycin and Teicoplanin MIC for all the Methicillin resistant isolates were tested by BMD method).(4)

The test includes S. aureus ATCC 29213 as negative control and S. aureus ATCC 700699 (MU 50) VISA Strain procured from Hi-Media as positive control. Interpretation was done based on CLSI Guidelines.

\section{RESULTS}

\begin{tabular}{|c|c|}
\hline Specimen & CoNS (n= 104) \\
\hline Pus & $\mathbf{7 2}$ \\
\hline Blood & 22 \\
\hline Body Fluid & 8 \\
\hline Urine & 2 \\
\hline \multicolumn{2}{|c|}{ Table 1. Specimen Wise distribution of CoNS Species } \\
$(\mathbf{n = 1 0 4 )})$
\end{tabular}

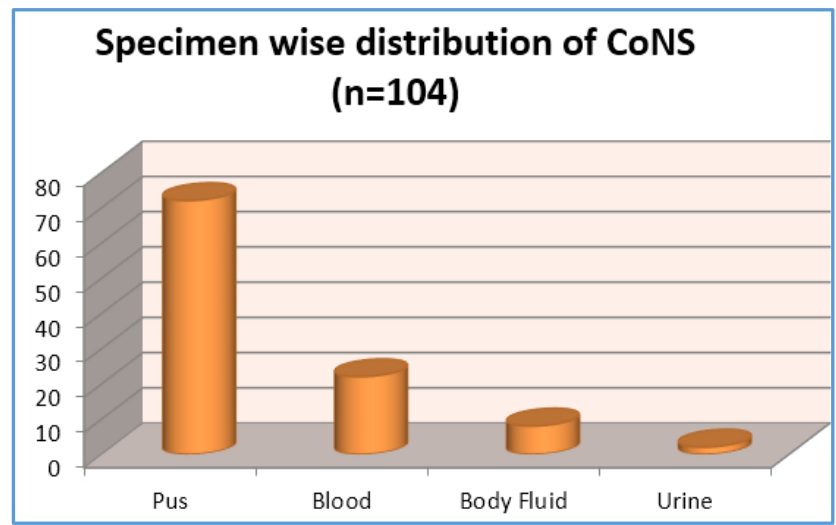

Among the samples 72 CoNS were isolated from pus, 22 were from blood and 8 were from body fluid and 2 from urine samples.

\begin{tabular}{|c|c|c|}
\hline Types & No. of Patients & Percentage \\
\hline MR CoNS & $\mathbf{2 8}$ & $26.92 \%$ \\
\hline MS CoNS & 76 & $73.08 \%$ \\
\hline Total & $\mathbf{1 0 4}$ & $\mathbf{1 0 0} \%$ \\
\hline Table 2. Distribution of MR CoNS and MS CoNS (n= 104) \\
\hline
\end{tabular}

\section{Distribution of MR CoNS and MS CoNS (\%)}

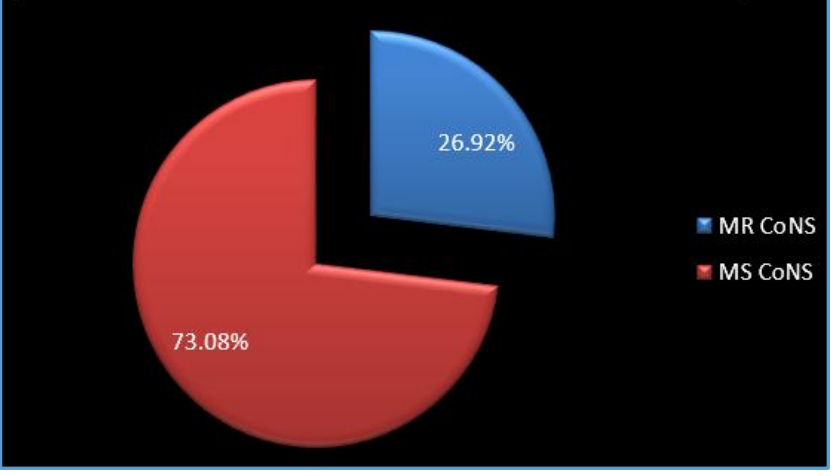


Among the 104 CoNS species, 72 were S. epidermidis, 19 were S. lugdunensis, 11 were S. haemolyticus, 2 were S.saprophyticus. Among them, 28 (26.92\%) were Methicillin Resistant CoNS (MRCoNS) and 76 (73.08\%) were Methicillin Sensitive CoNS (MSCoNS).

Among the 28 MRCoNS 19 were S. epidermidis, 6 were S.lugdunensis and 3 were S. haemolyticus species. The sample wise distribution of MRCoNS was analysed, which showed highest percentage of MRCoNS isolated in pus sample (20.19\%) followed by blood (4.81\%) and fluid (1.92\%).

The department wise isolation of Methicillin Resistant CoNS was analysed. It showed 9 were from surgery, 6 were from medicine, 7 from ortho, 4 from $O G$ and 2 from paediatric unit.

Phenotypic Detection of Glycopeptide Resistance among Methicillin Resistant Coagulase Negative Staphylococci $(n=28)$ was analysed.
Vancomycin screen agar (BHIA $6 \mu \mathrm{g}$ - vancomycin) showed the growth of 1 Methicillin resistant CoNS isolate.

The Vancomycin MIC of the MRCoNS isolates by E-test method was analysed. Among the 28 MRCoNS, 27 MRCoNS had MIC $\leq 4 \mu \mathrm{g} / \mathrm{mL}$, only one MRCoNS isolate had MIC between $8-16 \mu \mathrm{g} / \mathrm{mL}$ (VISE). The Teicoplanin MIC of MRCoNS isolates by E-test was analysed. All the 28 isolates were susceptible to Teicoplanin.

The Vancomycin MIC of the MRCoNS isolates by Broth microdilution method was analysed. Among the 28 MRCoNS 27 MRCoNS had MIC $\leq 4 \mu \mathrm{g} / \mathrm{mL}$, only one isolate had MIC between $8-16 \mu \mathrm{g} / \mathrm{mL}$ (VISE). The Teicoplanin MIC of MRCoNS isolates by Broth microdilution method was analysed. All the 28 isolates had MIC $\leq 8 \mu \mathrm{g} / \mathrm{mL}$ in the susceptible range.

\begin{tabular}{|c|c|c|c|c|}
\hline Isolate & No. of Isolate & Vancomycin MIC $(\boldsymbol{\mu g} / \mathbf{m L})$ & Teicoplanin MIC $(\boldsymbol{\mu g} / \mathbf{m L})$ & Specimen \\
\hline $\begin{array}{c}\text { S. epidermidis } \\
\text { (VISE) }\end{array}$ & 1 & 8 (Intermediate) & 4 (Susceptible) & Blood \\
\hline \multicolumn{3}{|c}{ Table 3. Details of Vancomycin Intermediate Staphylococcus epidermidis (VISE) Isolates } \\
\hline
\end{tabular}

\begin{tabular}{|c|c|c|c|c|c|c|c|c|c|c|c|c|}
\hline Isolate & P $10 \mu \mathrm{g}$ & ERY $15 \mu g$ & CX $30 \mu \mathrm{g}$ & CTX $30 \mu \mathrm{g}$ & CIP $5 \mu \mathrm{g}$ & $\begin{array}{c}\text { COT } \\
1.25 / 23.75 \mu \mathrm{g}\end{array}$ & $\begin{array}{c}\text { GM } \\
10 \mu \mathrm{g}\end{array}$ & $\begin{array}{l}\text { VAN } \\
\text { (MIC) }\end{array}$ & $\begin{array}{c}\text { TEI } \\
\text { (MIC) }\end{array}$ & \begin{tabular}{|c|}
$\mathrm{LZ}$ \\
$30 \mu \mathrm{g}$
\end{tabular} & $\begin{array}{l}\text { RIF } \\
5 \mu \mathrm{g}\end{array}$ & $\begin{array}{c}R P \\
15 \mu g\end{array}$ \\
\hline VISE & $\mathrm{R}$ & $\mathrm{R}$ & $\mathrm{R}$ & $\mathrm{R}$ & $\mathrm{R}$ & $\mathrm{R}$ & S & I & $S$ & S & S & S \\
\hline \multicolumn{13}{|c|}{ Table 4. Antibiogram of the VISE Isolates } \\
\hline
\end{tabular}

S- Susceptible, I- Intermediate, R- Resistant.

\section{Abbreviation}

P- Penicillin, ERY- Erythromycin, CX- Cefoxitin, CIPCiprofloxacin, COT- Trimethoprim/ Sulfamethoxazole, GMGentamicin, VAN- Vancomycin, TEI- Teicoplanin, LZLinezolid, RIF- Rifampin, RP- Quinupristin/ Dalfopristin.

Phenotypic detection of Glycopeptide resistance among Methicillin Resistant Staphylococci by various methods showed one isolate to be Vancomycin Intermediate S.epidermidis (VISE) from blood sample. This isolate was susceptible to Gentamicin, Teicoplanin, Linezolid, Rifampicin, Quinupristin/ Dalfopristin by reference CLSI methods.

\section{DISCUSSION}

Nowadays, CoNS has emerged as a pathogen causing serious nosocomial infections, particularly in immunocompromised patients and those suffering from chronic illnesses. It has been found that Methicillin resistance is more prevalent among CoNS that led to the increased use of glycopeptides as empirical therapy. In recent years, Glycopeptides Resistant CoNS (GRCoNS) have increasingly been reported. The emergence of resistance to Glycopeptides like Vancomycin and Teicoplanin in Staphylococci will have a significant impact on the treatment outcome. Hence, early detection of Glycopeptide resistance is of great clinical significance in combating resistance. In view of this above perspective, the current study was conducted to know the prevalence of Glycopeptide resistance in our geographical area by various phenotypic methods. A total of 104 Staphylococcus species were isolated from various clinical samples. The present study detected 28 (26.92\%) MRCoNS. Rachana Solanki et al (2012)(5) documented 59.2\% MRCoNS, Surekha Y Asangi et al (2011)(6) documented 67.7\% MRCoNS. The sample wise distribution of MRCoNS was analysed, which showed 21 (20.19\%) were from pus followed by blood (4.81\%). The department wise isolation of MRCoNS showed $32.14 \%$ were from surgery and $25 \%$ from orthopaedic wards.

Phenotypic Detection of Glycopeptide Resistance among Methicillin Resistant Staphylococci by Vancomycin Screen Agar

The phenotypic detection of Glycopeptide resistance by vancomycin screen agar (BHIA-6V) showed out of 28 only one (3.6\%) MRCoNS isolate grew on it. Dr. Dhanalakshmi TA, Umapathy BL et al (2012)(7) have stated that the Vancomycin screen agar test can be used as reliable screening method for detection of VRSA in their study from Karnataka, India. Gandham Pavani (2012)(8) has concluded in his study from Andhra Pradesh, India, that Vancomycin screen agar can be used as a method for Vancomycin resistant since it showed $100 \%$ specificity and $100 \%$ negative predictive value.

Phenotypic Detection of Glycopeptide Resistance among MRCoNS by E-Test Method and Broth Microdilution

Glycopeptide resistant detection of the 28 MRCoNS isolates by E-test method with vancomycin showed 27 (96.4\%) of isolates to be Vancomycin Susceptible CoNS with MIC $\leq 4$ $\mathrm{mcg} / \mathrm{mL}$. Only $1(3.6 \%)$ isolate was found to be VISE (Vancomycin intermediate S. epidermidis) with MIC $8 \mathrm{mcg} / \mathrm{mL}$. No VRCoNS was detected. E-test method with Teicoplanin showed all the MRCoNS isolates to be $100 \%$ susceptible.

The glycopeptides resistant detection with vancomycin by CLSI reference broth microdilution method showed 27 (96.4\%) of isolates to be Vancomycin Susceptible CoNS with MIC $\leq 4 \mathrm{mcg} / \mathrm{mL}$. Only $1(3.6 \%)$ isolate was found to be VISE 
with MIC $8 \mathrm{mcg} / \mathrm{mL}$. No VRCoNS was detected. BMD method with Teicoplanin showed all the MRCoNS isolates to be $100 \%$ susceptible.

The present study showed the prevalence of VISE to be 1 (3.6\%) among 28 MRCoNS strains. The MIC by E-test and BMD showed concordant results in this study.

\section{Various Studies showing the Prevalence of Glycopeptide Resistance among Coagulase Negative Staphylococci}

Evelina Tacconelli et al- 2001(9) Italy documented 3.73\% Glycopeptide resistant CoNS (among CoNS) by E-test method. Harekrishna Tiwari et al- 2006(10) Varanasi, India have reported $1.07 \%$ GRCoNS and $2.15 \%$ Glycopeptide intermediate CoNS (among CoNS) by Agar dilution method. GA Menzes and BN Harish et al-(11) 2008 Pondicherry, India have documented $23.8 \%$ vancomycin intermediate CoNS (among MRCoNS) by E-test method.

Silvia Natoli et al 2009(12) Italy documented 5.4\% CoNS with reduced susceptibility to glycopeptides (among CoNS) by E-test in blood samples of patients from haematology wards. Rachna Solanki et al 2012(5) Vadodara, India have shown 1.63\% VRCoNS (among MRCoNS) by E-test method.

$S$ Tevell et al 2013(13) Sweden documented 11.5\% Teicoplanin resistant CoNS (among S. epidermidis) by E-test method. Sunil B Bhamare et al 2014(14) Pune, India have documented 1.8\% VRCoNS (among MRCoNS) by Broth microdilution method. Xiao Xue Ma, En Hua Wang et al

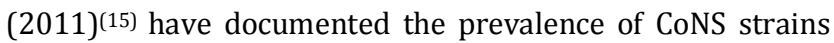
non-susceptible to Teicoplanin increased from $4.5 \%-6.7 \%$ between 2008 and 2009.

\section{The Present Study Detected 1 (3.6\%) VISE Isolate (among MRCoNS) by E-test and Broth Microdilution Method}

The present study showed the prevalence of Vancomycin Intermediate CoNS to be $3.6 \%$. This was concordant with the study conducted by Harekrishna Tiwari et al (2006),(10) who have documented $2.15 \%$ of Glycopeptide intermediate CoNS. In the present study, the VISE isolate was isolated from blood sample of a patient with infective endocarditis. This isolate was multidrug resistant but was susceptible to Gentamicin, Teicoplanin, Linezolid, Rifampicin, Quinupristin/ Dalfopristin by reference CLSI methods. Adina C Musta, Kathleen Riederer et al (2009)(16) have compared MIC by E-test method and standard microdilution method and found the results to be concordant for the most isolates. FW Goldstein, A Coutrot et al (1990)(17) have demonstrated that susceptibility of Coagulase negative staphylococci to Teicoplanin cannot be inferred from results of tests of vancomycin susceptibility and testing for Teicoplanin susceptibility should be routinely determined for patients treated with Teicoplanin.

\section{CONCLUSION}

Staphylococci is notorious for causing wide range of hospital and community acquired infection, because of the emergence of resistance to multiple antibiotics and limited therapeutic options. The prevalence of Vancomycin Intermediate Staphylococcus epidermidis (VISE) was 3.6\%. Early detection of these strains is crucial to establish an appropriate antimicrobial therapy, thereby reducing the mortality and morbidity associated with these infections and also to prevent hospital acquired infections. On comparison of various phenotypic methods, E-test can be used to detect Glycopeptide resistance since it is simple and easy to use despite its cost when compared to broth microdilution which is labour intensive and needs special training. Formulating antibiotic policy and following them, judicial use of higher antibiotics, strict aseptic precautions are the measures that can be taken to combat the serious therapeutic challenge faced with emerging multi-drug resistant Staphylococcus species.

\section{REFERENCES}

[1] Kloos WE, Schleifer KH. Simplified scheme for routine identification of human staphylococcus species. J Clin Microbiol 1975;1(1):82-8.

[2] Konemann EW, Allen SD, Janda WM, et al. The Gram positive cocci. Staphylococci and related organisms. In: Colour atlas and diagnosis microbiology. $6^{\text {th }}$ edn. 2006:623-71.

[3] Clinical and Laboratory Standards Insitute (CLSI). Performance standards for antimicrobial disc susceptibility tests. Approved standard - Eleventh edition. MO2 - A11, Wayne, PA, USA: 2014;34(1).

[4] Clinical and Laboratory standards Institute (CLSI). Methods for dilution antimicrobial susceptibility tests for bacteria that grow aerobically. Approved standard -Ninth edition. M07 - A9. Wayne, PA, USA: 2014;34(1).

[5] Solanki R, Javadekar TB. Incidence of vancomycin resistant staphylococci from various clinical isolates in a tertiary care hospital. National Journal of Laboratory Medicine 2012;1(1):23-5.

[6] Asangi SY, Mariraj J, Sathyanarayan MS, et al. Speciation of clinically significant Coagulase Negative Staphylococci and their antibiotic resistant patterns in a tertiary care hospital. Int J Biol Med Res 2011;2(3):735-9.

[7] Dhanalakshmi TA, Umapathy BL, Mohan DR. Prevalence of Methicillin, Vancomycin and Multidrug Resistance among Staphylococcus aureus. J Clin and Diagnostic Research 2012;6(6):974-7.

[8] Pavani G. Increased vancomycin resistance associated with decreased methicillin resistance in staphylococci. J Microbiol Biotech Res 2012;2(6):926-8.

[9] Tacconelli E, Tumbarello $M$, Donati KG, et al. Glycopeptide resistance among coagulase-negative staphylococci that cause bacteremia: epidemiological and clinical findings from a case-control study. Clinical Infectious Diseases 2001;33(10):1628-35.

[10] Tiwari HK, Sen MR. Emergence of vancomycin resistant Staphylococcus aureus (VRSA) from a tertiary care hospital from northern part of India. BMC Infectious Diseases 2006;6:156.

[11] Menezes GA, Harish BN, Sujatha S, et al. Emergence of vancomycin-intermediate Staphylococcus species in southern India. Journal of Medical Microbiology 2008;57(Pt 7):911-2.

[12] Natoli S, Fontana C, Favaro M, et al. Characterization of coagulase-negative staphylococcal isolates from blood with reduced susceptibility to glycopeptides and therapeutic options. BMC Infect Dis 2009;9:83. 
[13] Tevell S, Claesson C, Hellmark B, et al. Heterogeneous glycopeptide intermediate Staphylococcus epidermidis isolated from prosthetic joint infections. Eur J Clin Microbiol Infect Dis 2014;33(6):911-7.

[14] Bhamare SB, Karmarkar A, Iyer V, et al. Study of prevalence of methicillin and vancomycin resistance in multidrug resistant coagulase negative staphylococci. International J of Healthcare and Biomedical Research 2014;2(3):67-72.

[15] Ma XX, Wang EH, Liu Y, et al. Antibiotic susceptibility of coagulase-negative staphylococci (CoNS): emergence of Teicoplanin-non-susceptible CoNS strains with inducible resistance to vancomycin. Journal of Medical Microbiology 2011;60(Pt 11):16618.
[16] Musta AC, Riederer K, Shemes S, et al. Vancomycin MIC plus heteroresistance and outcome of methicillinresistant Staphylococcus aureus bacteremia: trends over 11 Years. J Clin Microbiol 2009;47(6):1640-4.

[17] Goldstein FW, Coutrot A, Sieffer A, et al. Percentages and distributions of teicoplanin- and vancomycinresistant strains among coagulase-negative staphylococci. Antimicrob Agents Chemother 1990;34(5):899-900. 